\title{
RESPUESTA DE CANOLA PARA FORRAJE A LA DENSIDAD DE POBLACIÓN
}

\author{
RESPONSE OF CANOLA FORAGE TO PLANT DENSITY
}

\author{
David G. Reta-Sánchez, J. Santos Serrato-Corona², Héctor M. Quiroga-Garza, \\ Uriel Figueroa-Viramontes ${ }^{1}$, Arturo Gaytán-Mascorro ${ }^{1}$ y J. Antonio Cueto-Wong ${ }^{1}$
}

\begin{abstract}
'Campo Experimental La Laguna, Instituto Nacional de Investigaciones Forestales, Agrícolas y Pecuarias (INIFAP). Blvd. José Santos Valdez 1200. 27440, Col. Centro, Cd. Matamoros, Coahuila, México. Tel. 01-800-088-2222 Ext. 82415. Facultad de Agricultura y Zootecnia, Universidad Juárez del Estado de Durango. Domicilio Conocido, Ej. Venecia, Dgo. Apdo. Postal 1-142. Gómez Palacio, Durango, México.
\end{abstract}

*Autor de correspondencia (reta.david@inifap.gob.mx)

\section{RESUMEN}

El aumento en la densidad de población en canola (Brassica napus L. var. oleifera) para forraje puede incrementar los rendimientos de materia seca (MS) y nutrientes. Se realizaron dos experimentos para evaluar el efecto de la densidad de población sobre la composición química del forraje y los rendimientos de MS, proteína cruda (PC) y energía neta para lactancia $\left(\mathrm{EN}_{\llcorner}\right)$ en canola. Los experimentos se hicieron en Matamoros, Coahuila, México durante los ciclos 2011-2012 y 2012-2013. Las densidades de población a la cosecha fluctuaron entre 19.1 y 248 plantas $\mathrm{m}^{-2}$. La asociación entre la densidad de población y las variables de respuesta se analizó mediante regresión lineal simple $(\mathrm{P} \leq 0.05)$. El aumento en la densidad de plantas incrementó la concentración de PC y EN en el primer ciclo, mientras que en el segundo la composición química no fue afectada. En las bajas densidades, la plasticidad de la canola no compensó el rendimiento de MS, PC y EN por hectárea, ya que estas variables mostraron una relación lineal positiva $(P \leq$ 0.05 ) con la densidad de población. El rendimiento de MS fluctuó de 6,067 a 9,356 $\mathrm{kg} \mathrm{ha}^{-1}$. Los rendimientos de PC y EN se ajustaron a una función lineal $(P \leq 0.05)$. En el segundo año, los rendimientos de $P C(1,398$ a 2,260 $\left.\mathrm{kg} \mathrm{ha}^{-1}\right)$ y $\mathrm{EN}_{\mathrm{L}}\left(29,248\right.$ a $\left.54,487 \mathrm{MJ} \mathrm{ha}^{-1}\right)$ fueron mayores que los del primero, con 842 a 2,159 $\mathrm{kg} \mathrm{ha}^{-1}$ en PC y 17,533 a 44,617 $\mathrm{MJ} \mathrm{ha}^{-1}$ en $\mathrm{EN}_{\mathrm{L}}$. Los resultados indican que los rendimientos de MS y nutrientes en canola para forraje se incrementaron al aumentar la densidad de población, sin afectar la composición química del forraje.

Palabras clave: Brassica napus var. oleifera, composición química, rendimiento de materia seca, nutrientes.

\section{SUMMARY}

Increasing plant density in canola forage (Brassica napus L. var. oleifera) may improve dry matter (DM) and nutrient yields. Two experiments were conducted to evaluate the effect of plant density on forage chemical composition and yields of DM, crude protein (CP) and net energy for lactation $\left(\mathrm{NE}_{\mathrm{L}}\right)$ in canola. The trials were carried out during the 2011-2012 and 20122013 cycles in Matamoros, Coahuila, Mexico. Plant densities at harvest varied from 19.1 to 248 plants $\mathrm{m}^{-2}$. The association between plant density and the response variables were analyzed using simple linear regression $(P \leq 0.05)$. The increase in plant density enhanced the concentration of CP and NEL in the first cycle, whereas in the second cycle chemical composition was not affected. At low plant densities, the plasticity of canola did not compensate $\mathrm{DM}, \mathrm{CP}$ and $\mathrm{NE}_{L}$ yields, as these variables showed a positive linear relationship $(\mathrm{P} \leq 0.05)$ with plant density. Dry matter yield ranged from 6067 to $9356 \mathrm{~kg} \mathrm{ha}$ 1. Yields of $C P$ and $N E_{1}$ were fitted to a linear function $(P \leq 0.05)$. Yields of $C P$ (1398 to $\left.2260 \mathrm{~kg} \mathrm{ha}^{-1}\right)$ and $\mathrm{NE}_{\mathrm{L}}\left(29,248\right.$ to $\left.54,487 \mathrm{MJ} \mathrm{ha}^{-1}\right)$ in the second year were higher than those observed in the first year, with 842 to $2159 \mathrm{~kg} \mathrm{ha}^{-1}$ of $\mathrm{CP}$ and 17,533 to $44,617 \mathrm{MJ} \mathrm{ha}^{-1}$ of $\mathrm{NE}_{\mathrm{L}}$. Results indicate that $\mathrm{DM}$ and nutrient yields in canola forage increased as plant density increased, without affecting forage chemical composition.

Index words: Brassica napus var. oleifera, chemical composition, dry matter and nutrients yield.

\section{INTRODUCCIÓN}

La producción intensiva de ganado bovino lechero es una de las principales actividades económicas en el nortecentro de México. La alimentación del ganado se basa en forrajes como alfalfa (Medicago sativa L.), maíz (Zea mays L.), sorgo (Sorghum bicolor L. Moench.) y avena (Avena sativa L.) producidos bajo irrigación. Sin embargo, problemas como baja disponibilidad de agua, salinidad en el suelo, altas temperaturas ambientales y un reducido número de especies forrajeras limitan la producción de forraje en esta región (Reta-Sánchez et al., 2008), lo que ha obligado a la búsqueda de nuevas alternativas forrajeras.

La canola (Brassica napus L.) puede ser una buena alternativa para la producción de forraje en las regiones áridas y semiáridas de México, ya que produce forraje nutritivo con mayor eficiencia en el uso del agua que la avena, uno de los principales forrajes en la región (Reta et al., 2008; Reta et al., 2010). La canola posee algunas ventajas para la región como tolerancia a bajas temperaturas (Sovero, 1993) y salinidad (Francois, 1994) además de ser precoz para producir forraje (Reta et al., 2008).

La producción eficiente de forraje de canola aún requiere del desarrollo de tecnología para el manejo del cultivo. Una de las prácticas agronómicas importantes es la densidad de población, ya que ésta afecta significativamente el crecimiento de la planta y los costos de producción de forraje. La respuesta de la canola para semilla a la densidad de población está ampliamente documentada; sin embargo, ésta varía de acuerdo con las condiciones ambientales y manejo agronómico (Angadi et al., 2003; Morrison et al., 1990; Ortegón et al., 2002). La planta de 
canola para producción de semilla presenta una alta plasticidad bajo un amplio rango de densidades de población (de 20 a 80 plantas $\mathrm{m}^{-2}$ ) cuando la disponibilidad de agua es favorable (Angadi et al., 2003; Ortegón-Morales et al., 2007).

La expresión de la plasticidad en canola requiere de un ciclo de crecimiento largo, como el observado por Ortegón-Morales et al. (2007) en Tamaulipas, México (130 d). Cuando el ciclo de crecimiento es corto, la canola no tiene el tiempo suficiente para expresar totalmente la plasticidad en bajas densidades, por lo que el cultivo requiere una mayor densidad de población para obtener los mayores rendimientos (Angadi et al., 2003).

Bajo estas condiciones de crecimiento se encuentra la canola para forraje en la Comarca Lagunera, donde se observan ciclos de crecimiento entre 67 y 107 d, de acuerdo con la fecha de siembra y etapa de cosecha (Reta et al., 2008; Reta et al., 2010; Reta et al., 2015). El objetivo del presente estudio fue determinar la respuesta de la canola a la densidad de población en términos de la composición química del forraje y los rendimientos de materia seca (MS), proteína cruda $(\mathrm{PC})$ y energía neta para lactancia $\left(\mathrm{EN}_{\llcorner}\right)$.

\section{MATERIALES Y MÉTODOS}

El estudio se realizó en un campo experimental localizado en Matamoros, Coahuila, México (25 $32^{\prime} \mathrm{N}, 103^{\circ} 14^{\prime}$ $\mathrm{O}$ y altitud de $1150 \mathrm{~m}$ ), en un suelo arcillo-limoso. La preparación de la cama de siembra se realizó con un paso de arado a $0.30 \mathrm{~m}$ de profundidad, seguido de doble rastreo y nivelación. Antes de la siembra, cada parcela experimental se fertilizó en forma manual con $70 \mathrm{~kg} \mathrm{~N} \mathrm{ha}^{-1}$ (urea) y 100 $\mathrm{kg}$ de $\mathrm{P}_{2} \mathrm{O}_{5}$ ha $^{-1}$ (fosfato monoamónico).

La siembra se hizo manualmente en suelo seco el 17 de diciembre de 2011 y el 7 de octubre de 2012. El siguiente día después de la siembra se aplicó un riego con una lámina de $150 \mathrm{~mm}$. Doce días después de la siembra (dds) se aplicó un riego ligero (60 mm de lámina) para facilitar la emergencia de plántulas. Se utilizó la canola de primavera (Brassica napus L. var. oleifera) variedad IMC 205 (Inter. Mountain Cargill). El área experimental fue irrigada con el método de riego superficial mediante un sistema de tubos de plástico con compuertas.

Se evaluaron cinco densidades de población en un diseño experimental de bloques completos al azar con cuatro repeticiones. Los tratamientos establecidos en el inicio del experimento fueron: 60, 105, 150, 195 y 240 plantas $\mathrm{m}^{-2} \mathrm{en}$ 2011-2012; y 20, 70, 120, 170 y 220 plantas $\mathrm{m}^{-2}$ en 20122013. Las parcelas experimentales fueron de 8 surcos con un distanciamiento de $0.38 \mathrm{~m}$ y $5 \mathrm{~m}$ de longitud. Las me- diciones se hicieron en los tres surcos centrales de cada parcela con una longitud de $3 \mathrm{~m}\left(3.42 \mathrm{~m}^{2}\right)$.

De acuerdo con las densidades de población evaluadas, en cada parcela se utilizó una densidad de siembra superior en $50 \%$ a la densidad de población correspondiente, y posteriormente a los 25 dds se realizó un aclareo de plantas para dejar la densidad a evaluar. La cosecha se llevó a cabo a los 102 dds en 2011-2012, y a los 97 dds en 2012-2013, cuando el cultivo alcanzó las etapas de floración completa (etapa 4.4) y 50 \% de floración (etapa 4.2) en el primero y segundo año, respectivamente (Harper y Berkenkamp, 1975).

Durante el ciclo de crecimiento se aplicaron dos riegos, a los 53 y 77 dds en 2011-2012 y a los 45 y 67 dds en 2012-2013. En cada año, inmediatamente antes del primer riego, se aplicaron $120 \mathrm{~kg} \mathrm{~N}^{-1}$ con sulfato de amonio granulado. El control de maleza se hizo en forma manual con azadón. En 2011-2012 los valores promedio de temperatura máxima, temperatura mínima y humedad relativa fueron $25.1^{\circ} \mathrm{C}, 6.8^{\circ} \mathrm{C}$ y $34.9 \%$, respectivamente; mientras que en $2012-2013$ estos valores fueron $25.7^{\circ} \mathrm{C}, 9.8^{\circ} \mathrm{C}$ y $46.4 \%$, respectivamente. La precipitación y evaporación potencial acumulados en el primer ciclo fueron $11.4 \mathrm{~mm}$ y $429.2 \mathrm{~mm}$, respectivamente; mientras que en el segundo la precipitación fue de $23.6 \mathrm{~mm}$ y la evaporación potencial fue de $467.4 \mathrm{~mm}$.

En la cosecha se determinaron los rendimientos de forraje fresco y de MS. El contenido de MS se determinó en una muestra de $1.14 \mathrm{~m}^{2}$ tomada al azar de la muestra usada para las mediciones. Para ello se muestreó uno de los surcos centrales de cada parcela de $3 \mathrm{~m}$ de longitud. Las plantas muestreadas fueron secadas a $60^{\circ} \mathrm{C}$ en una estufa de aire forzado hasta alcanzar peso constante. El rendimiento de MS se determinó multiplicando el rendimiento de forraje fresco por el contenido de MS de cada parcela.

La distribución de MS en los órganos de la parte aérea se determinó con una muestra al azar de $0.57 \mathrm{~m}^{2}$ tomada de la parcela usada para las mediciones; para este propósito se tomó una muestra de $1.5 \mathrm{~m}$ de longitud de uno de los surcos centrales. Después de separar las plantas en tallos, hojas (lámina y pecíolos) y órganos reproductivos (flores y silicuas), se secaron a $60^{\circ} \mathrm{C}$ hasta alcanzar peso constante para determinar el porcentaje de MS asignado a cada órgano de la parte aérea del cultivo.

Las plantas muestreadas para estimar el contenido de MS fueron también usadas para determinar la composición química del forraje en términos de proteína cruda (PC), fibra detergente ácido (FDA), fibra detergente neutro (FDN) y energía neta para lactancia $\left(E N_{L}\right)$. Las plantas fueron molidas con 
un molino Wiley® (Thomas Scientific, Swedesboro, NJ, USA) con malla de $1 \mathrm{~mm}$. Las muestras fueron analizadas de acuerdo con el procedimiento descrito por Goering y Van Soest (1970) para FDN y FDA, y con el método Kjeldahl para N (Bremner, 1996). El contenido de EN se estimó de acuerdo con las metodologías del Consejo Nacional de Investigación (NRC, 2001). Los rendimientos de PC y EN por hectárea se obtuvieron al multiplicar los contenidos de PC y EN por el rendimiento de MS de cada parcela experimental.

Se hicieron análisis de regresión lineal simple $(P \leq 0.05)$ para determinar la relación entre las siguientes variables: densidad de población con contenidos de PC, FDN y EN ; y de la densidad de población con los rendimientos de MS, PC y EN $\mathrm{L}_{\mathrm{L}}$ Los análisis estadísticos de las variables evaluadas se presentan con los datos combinados de los dos años y por año, de acuerdo con las respuestas observadas en los análisis de regresión realizados. El análisis de la información se efectuó con el programa estadístico SAS (SAS Institute 1989).

\section{RESULTADOS Y DISCUSIÓN}

\section{Composición química del forraje}

El efecto de la densidad de población sobre la composición química del forraje fue diferente en los dos años del estudio. En el primer año, el aumento de la densidad de población mejoró la composición química del forraje, mientras que en el segundo la composición química no fue afectada. Este comportamiento se relacionó con una etapa del desarrollo más temprana en altas densidades de población en el primer año, lo cual se reflejó en una reducción de la distribución porcentual de MS en órganos reproductivos de 12.0 a $7.0 \%$ en las mayores densidades.

En el ciclo 2011-2012 se encontró una relación lineal positiva $(P \leq 0.05)$ entre la densidad de población y los contenidos de PC y EN en el forraje, la cual explicó $54.93 \%$ y $45.51 \%$ de la variabilidad observada para PC y EN ${ }_{L}$, respectivamente. En el ciclo 2012-2013 la densidad de población no modificó significativamente los contenidos de las dos variables $(P>0.05)$. En la concentración de FDN no se encontró una relación significativa $(P>0.05)$ con el aumento de población en los dos años de estudio (Figura 1).

La composición química del forraje fue mayor en el segundo año, con contenidos de $245.9 \mathrm{~g} \mathrm{~kg}^{-1}$ de PC y 5.48 $\mathrm{MJ} \mathrm{kg}{ }^{-1} \mathrm{MS}$ de $\mathrm{EN}_{\llcorner}$, y menor concentración de FDN (397.0 $\left.\mathrm{g} \mathrm{kg}^{-1}\right)$ con respecto al primer año, donde alcanzó valores de $193.8 \mathrm{~g} \mathrm{~kg}^{-1}, 510.3 \mathrm{~g} \mathrm{~kg}^{-1}$ y $3.94 \mathrm{MJ} \mathrm{kg}^{-1} \mathrm{MS}$ de PC, FDN y EN, respectivamente (Figura 1). La mejor composición química en el segundo año se debió a la etapa del desarro- llo más temprana en la que se cosechó, 50 \% de floración con respecto a floración completa en el primer ciclo.

El resultado indica una mayor distribución de MS en la hoja (44.4\%) y menor acumulación en el tallo (53.3 \%) con respecto a los valores observados en el primer año con 32.0 y $58.2 \%$ para hoja y tallo, respectivamente. Este comportamiento puede explicarse por el mayor contenido de PC en hojas (200.0 a $250.0 \mathrm{~g} \mathrm{~kg}^{-1}$ ) con respecto al tallo $\left(100.0 \mathrm{~g} \mathrm{~kg}^{-1}\right)$ de acuerdo con lo observado por Rao y Horn (1995); y la menor concentración de FDN en hoja (208.0 $\mathrm{g} \mathrm{kg}^{-1}$ ) con respecto a la del tallo $\left(480.0 \mathrm{~g} \mathrm{~kg}^{-1}\right)$ como lo reportaron Chapman et al. (2009).

La mejor composición química aquí registrada fue similar a la observada por Reta et al. (2010) en la misma localidad, con valores de $230 \mathrm{~g} \mathrm{~kg}^{-1}$ para PC, $398 \mathrm{~g} \mathrm{~kg}^{-1}$ para FDN y $5.69 \mathrm{MJ} \mathrm{kg}^{-1}$ para $\mathrm{EN}_{\mathrm{L}}$. En relación con otros estudios realizados en canola, la concentración de PC obtenida en el estudio (169.3 a $272.8 \mathrm{~g} \mathrm{~kg}^{-1}$ ) fue similar o mayor a la encontrada (126 a $230 \mathrm{~g} \mathrm{~kg}^{-1}$ ) en estudios previos (Rao y Horn, 1995; Sincik et al., 2007; Wiedenhoeft y Barton, 1994), variación debida a las condiciones ambientales, manejo y edad del cultivo.

\section{Rendimientos de materia seca y nutrientes}

Con los datos combinados de los dos años evaluados se encontró una relación lineal positiva $(P \leq 0.05)$ entre el número de plantas por $\mathrm{m}^{2}$ y el rendimiento de MS. En esta relación el incremento del rendimiento de MS fue de 718.6 $\mathrm{kg} \mathrm{ha}^{-1}$ por cada aumento de 50 plantas $\mathrm{m}^{-2}$ en la densidad. En el intervalo evaluado de 19.1 a 248 plantas $\mathrm{m}^{-2}$, el rendimiento de MS fluctuó de $6067 \mathrm{~kg} \mathrm{ha}^{-1}$ en la densidad baja a $9356 \mathrm{~kg} \mathrm{ha}^{-1}$ en la densidad alta (Figura 2). Los rendimientos de MS estimados a partir de la densidad de 155 plantas $\mathrm{m}^{-2}$ fueron similares a los encontrados en trabajos previos realizados en la Comarca Lagunera con densidades entre 180 y 200 plantas $\mathrm{m}^{-2}$, en los cuales se alcanzaron rendimientos de MS de 8000 a 10,000 kg ha-1 (Cruz-Chairez et al., 2012; Reta et al., 2008; Reta et al., 2010).

Los rendimientos de PC y EN $\mathrm{L}_{\llcorner}$también se ajustaron a una función lineal $(P \leq 0.05)$ en respuesta a la densidad de población; sin embargo, cada año se ajustó a una regresión diferente. En el segundo año los rendimientos de PC (1398 a $\left.2260 \mathrm{~kg} \mathrm{ha}^{-1}\right)$ y EN $\left(29,248\right.$ a 54,487 $\left.\mathrm{MJ} \mathrm{ha}^{-1}\right)$ fueron mayores que los obtenidos en el primero, los cuales fueron de 842 a $2159 \mathrm{~kg} \mathrm{ha}^{-1}$ de PC y 17,533 a 44,617 MJ ha-1 en $\mathrm{EN}_{\mathrm{L}}$.

Por cada incremento de 50 plantas $\mathrm{m}^{-2}$, el rendimiento de PC aumentó en 236.7 y $171.6 \mathrm{~kg} \mathrm{ha}^{-1}$ en 2011-2012 y 2012-2013, respectivamente; en $\mathrm{EN}_{\mathrm{L}}$ el aumento fue de 

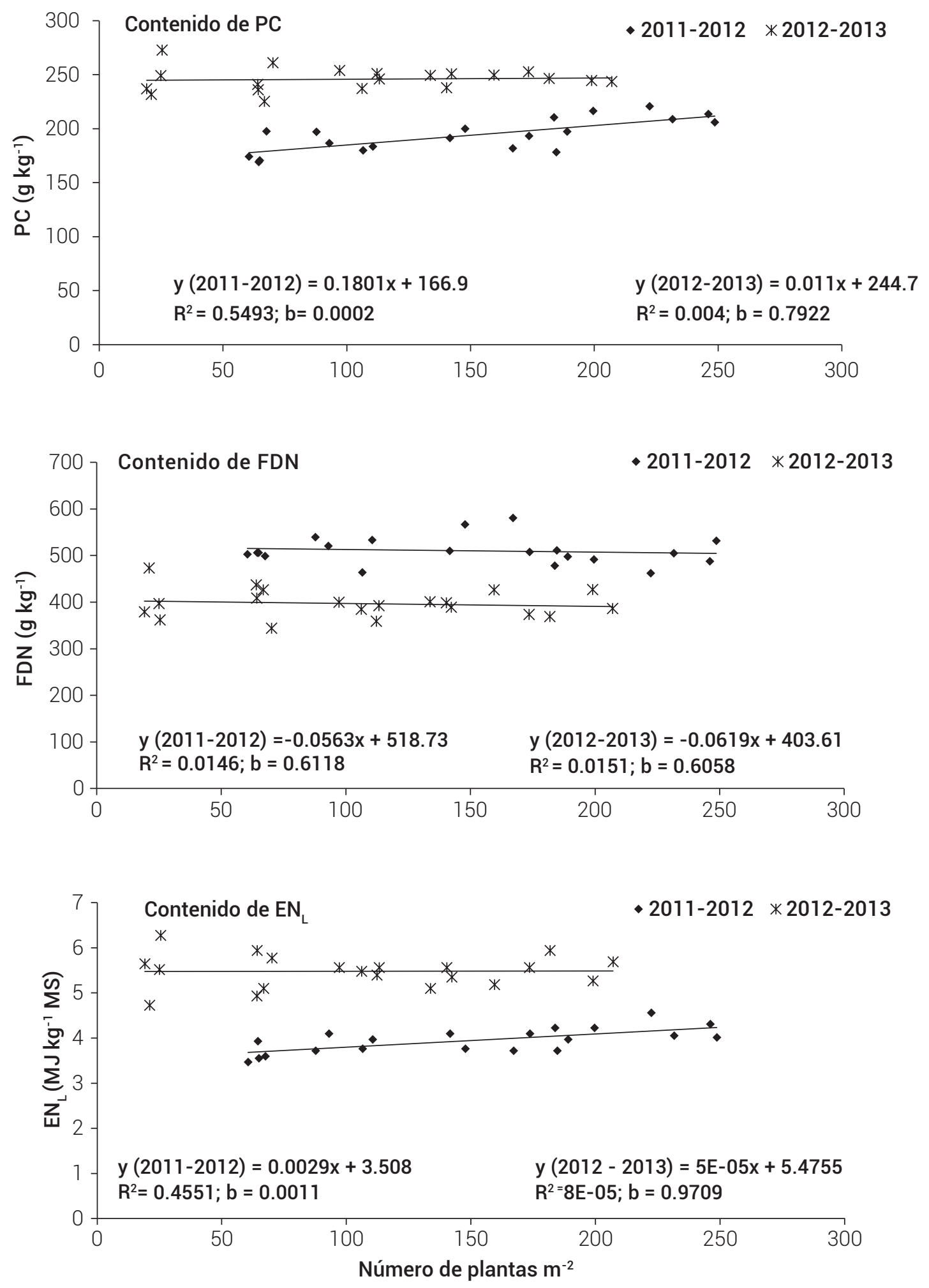

Figura 1. Contenidos de proteína cruda (PC), fibra detergente neutro (FDN) y energía neta para lactancia $\left(\mathrm{EN}_{\mathrm{L}}\right)$ de canola para forraje en relación al número de plantas $\mathrm{m}^{-2}$ cosechadas durante los ciclos 2011-2012 y 2012-2013 en Matamoros, Coahuila, México. $M S=$ materia seca; $b=p$-valor de la pendiente. 

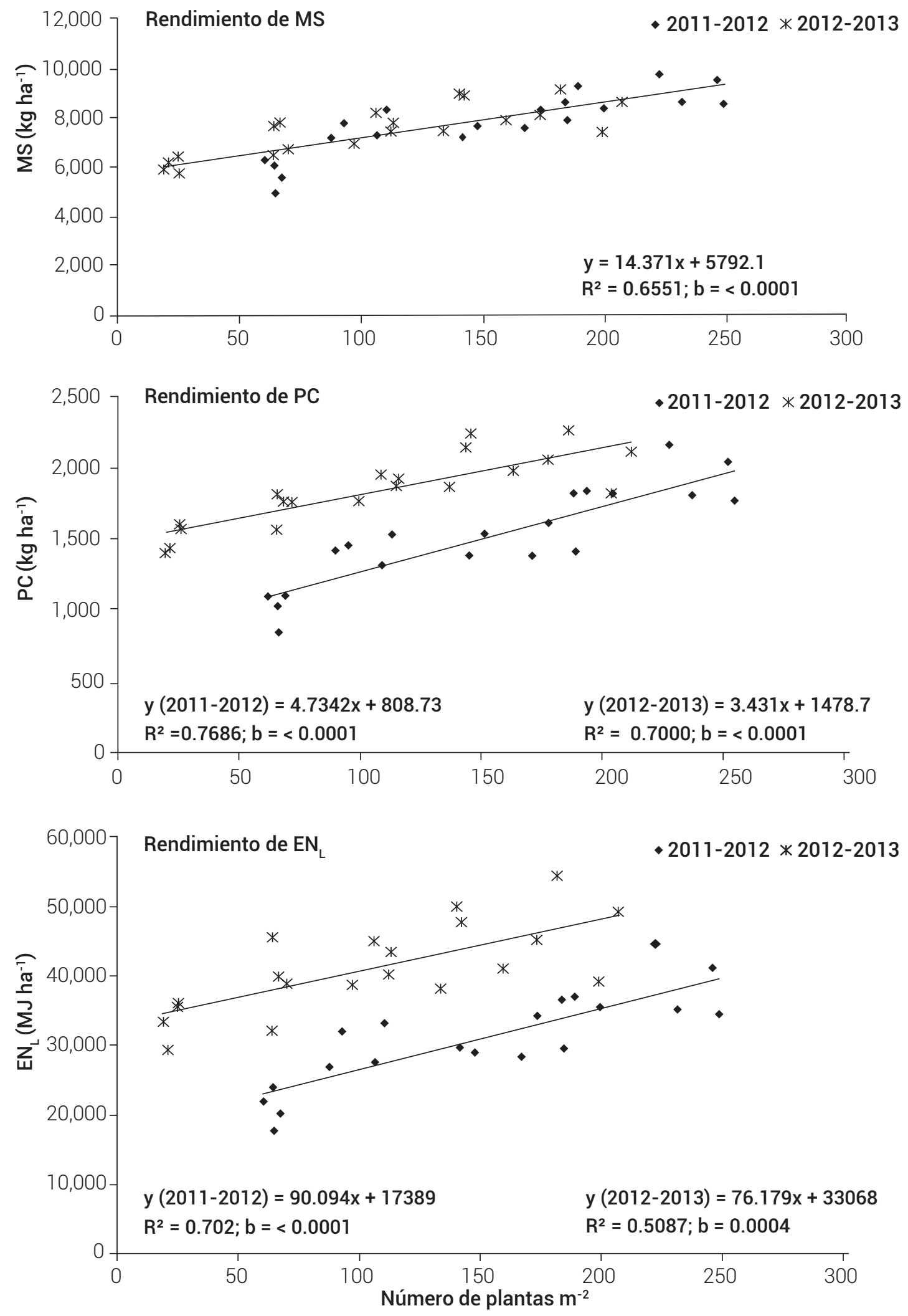

Figura 2. Rendimientos de materia seca (MS), proteína cruda (PC) y energía neta para lactancia $\left(E N_{L}\right)$ de canola para forraje en relación al número de plantas $\mathrm{m}^{-2}$ cosechadas durante los ciclos 2011-2012 y 2012-2013 en Matamoros, Coahuila, México. $b=p$-valor de la pendiente. 
$4505 \mathrm{MJ} \mathrm{ha}^{-1}$ en el primer año, y de $3809 \mathrm{MJ} \mathrm{ha}^{-1}$ en el segundo (Figura 2). En el primer año la respuesta a la densidad de población se relacionó tanto con el incremento en los contenidos de nutrientes como con el rendimiento de MS, con mayor grado de asociación en el segundo caso. En el segundo año, el incremento en los rendimientos de PC y EN $\mathrm{L}_{\mathrm{L}}$ se asoció sólo con el mayor rendimiento de MS, ya que los contenidos de estos nutrientes no fueron afectados por la densidad de población.

En otros trabajos con canola para forraje realizados en la Comarca Lagunera y establecidos con densidades de 180 a 200 plantas $\mathrm{m}^{-2}$ y en surcos a 0.20 y $0.38 \mathrm{~m}$, se han obtenido rendimientos de PC de 1501 a $2475 \mathrm{~kg} \mathrm{ha}^{-1}$ y de 42,175 a 62,057 $\mathrm{MJ} \mathrm{ha}^{-1}$ en EN (Cruz et al., 2012; Reta et al., 2008; Reta et al., 2015).

La respuesta de la canola en rendimientos de MS, PC y $E N_{L}$ muestra que no se presentó la plasticidad observada en estudios con densidades de población para producción de semilla, en los cuales la canola puede compensar la baja densidad de población con la formación de un mayor número de silicuas por planta (Angadi et al., 2003; Ortegón et al., 2002). El grado de plasticidad de la canola depende de la disponibilidad de recursos como luz, agua y nutrientes, además de que requiere más tiempo para la producción y desarrollo de ramas (Angadi et al., 2003).

En el presente estudio la canola tuvo los recursos suficientes para su desarrollo; sin embargo, su periodo de crecimiento fue corto ya que se cosechó a los 102 y 97 dds, cuando el cultivo alcanzó las etapas de floración completa y 50 \% de floración en el primero y segundo año, respectivamente. Debido a esto el cultivo requirió las densidades de población más altas para obtener los mayores rendimientos de MS y nutrientes; respuesta que fue similar a la observada por Angadi et al. (2003) en el rendimiento de semilla en canola establecida en regiones con un ciclo de crecimiento corto.

\section{CONCLUSIONES}

La canola para forraje mostró una baja plasticidad a la densidad de población en rendimientos de materia seca, proteína cruda y energía neta para lactancia debido a su ciclo de crecimiento corto; bajo estas condiciones los máximos rendimientos de materia seca y nutrientes se obtuvieron con las mayores densidades de población evaluadas en el estudio (207 a 248 plantas m²), sin afectar la composición química del forraje.

\section{AGRADECIMIENTOS}

Al Instituto Nacional de Investigaciones Forestales, Agrícolas y Pecuarias y Fundación Produce Coahuila, A.C. por el financiamiento de este estudio.

\section{BIBLIOGRAFÍA}

Angadi S. V., H. W. Cutforth, B. G. McConkey and Y. Gan (2003) Yield adjustment by canola grown at different plant populations under semiarid conditions. Crop Science 43:1358-1366.

Bremner J. M. (1996) Nitrogen-total. In: Methods of Soil Analysis. Part 3. Chemical Methods. D. L. Sparks (ed.). SSSA Book Series 5. Soil Science Society of America. Madison, WI. pp:1085-1121.

Chapman G., E. Bork, N. Donkor and R. Hudson (2009) Yields, quality and suitability of four annual forages for deer pasture in North Central Alberta. The Open Agriculture Journal 3:26-31.

Cruz C., J. J., G. Núñez H., R. Faz C., D. G. Reta S. y H. A. Serrato M. (2012) Potencial forrajero y eficiencia de uso del agua de canola (Brassica napus L.) en comparación con cultivos tradicionales en el ciclo de invierno. Agrofaz 12:125-130.

Francois L. E. (1994) Growth, seed yield, and oil content of canola grown under saline conditions. Agronomy Journal 86:233-237.

Goering H. K. and P. J. van Soest (1970) Forage fiber analyses (Apparatus, reagents, procedures, and some applications). Agriculture Handbook No. 379. ARS-USDA. Washington, DC. 20 p.

Harper F. R. and B. Berkenkamp (1975) Revised growth-stage key for Brassica campestris and B. napus. Canadian Journal of Plant Science 55:657-658.

Morrison M. J., P. B. E. McVetty and R. Scarth (1990) Effect of row spacing and seed rates on summer rape in southern Manitoba. Canadian Journal of Plant Science 70:127-137.

NRC-National Research Council (2001) Nutrient Requirements of Dairy Cattle. $7^{\text {th }}$ ed. National Academy Press, Washington, D.C. 408 p.

Ortegón M., A. S., A. Díaz F. y A. Rodríguez C. (2002) Rendimiento de híbridos de canola (Brassica napus L.) en diferentes métodos de siembra. Agricultura Técnica en México 28:151-158.

Ortegón-Morales A. S., A. Díaz-Franco y J. González-Quintero (2007) Cultivares de canola y su interacción con el ambiente y el método de siembra. Universidad y Ciencia 23:21-27.

Rao S. C. and F. P. Horn (1995) Cereals and brassicas for forage. In: Forages. R. F. Barnes, D. A. Miller and C. J. Nelson (eds.). lowa State University Press. Ames, lowa. pp:451-462.

Reta S., D. G., U. Figueroa V., R. Faz C., G. Núñez H., A. Gaytán M., J. S. Serrato C. y J. A. Payán G. (2010) Sistemas de producción de forraje para incrementar la productividad del agua. Revista Fitotecnia Mexicana 33 (Núm. Especial 4):83-87.

Reta S., D. G., J. S Serrato C., R. Figueroa V., J. A Cueto W., S. Berumen P. y J. Santamaría C. (2008) Cultivos alternativos con potencial de uso forrajero en la Comarca Lagunera. Libro Técnico Núm. 3. INIFAP-CIRNOC-CELALA. Torreón, Coah. 268 p.

Reta S., D. G., U. Figueroa V., J. S. Serrato C., H. M. Quiroga G., A. Gaytán M. y J. A. Cueto W. (2015) Potencial forrajero y productividad del agua en patrones de cultivos alternativos. Revista Mexicana de Ciencias Pecuarias 6:153-170.

SAS Institute (1989) SAS User's Guide. Statistics, Version 6.0. 4th. ed. SAS Institute Inc. Cary, NC.

Sincik M., U. Bilgili, A. Uzun and E. Acikgoz (2007) Short communication. Harvest stage effects on forage yield and quality for rape and turnip genotypes. Spanish Journal of Agricultural Research 5:510-516.

Sovero M. (1993) Rapeseed, a new oilseed crop for the United States. In: New Crops. J. Janick and J. E. Simon (eds.). Wiley. New York. pp:302-307.

Wiedenhoeft M. H. and B. A. Barton (1994) Management and environment effects on Brassica forage quality. Agronomy Journal 86:227232. 